\title{
Morfologia e biometria do timo em avestruzes (Struthio camelus)
}

\author{
Cheston Cesar Honorato Pereira ${ }^{1 *}$ \\ Márcia Rita Fernandes Machado ${ }^{2}$ \\ Angelita das graças de Oliveira Honorato ${ }^{3}$ \\ Bruno Gomes Vasconcelos ${ }^{4}$ \\ Thales Dias do Prado ${ }^{5}$ \\ ${ }^{1}$ Programa de Pós Graduação em Medicina Veterinária \\ Universidade Estadual Paulista "Júlio de Mesquita Filho", Campus Jaboticabal \\ Via de acesso Prof. Paulo Donato Castellane s/n, CEP 14884-900, Jaboticabal - SP, Brasil \\ ${ }^{2}$ Faculdade de Medicina Veterinária \\ Universidade Estadual Paulista "Júlio de Mesquita Filho", Jaboticabal - SP, Brasil \\ ${ }^{3}$ Faculdade de Medicina Veterinária, Universidade Federal de Uberlândia, Uberlândia - MG, Brasil \\ ${ }^{4}$ Faculdade de Medicina Veterinária, Universidade Federal do Mato Grosso, Sinop - MT, Brasil \\ ${ }^{5}$ Faculdade de Medicina Veterinária, Universidade de Rio Verde, Rio Verde - GO, Brasil \\ * Autor para correspondência \\ cheston@bol.com.br
}

\section{Resumo}

Estudou-se a anatomia do timo em 30 filhotes de avestruzes, 13 machos e 17 fêmeas com idade variando entre 10 a 20 dias, mediante fixação de cada ave em solução aquosa de formol a 10\%. As cadeias de lobos tímicos localizaram-se no terço distal da região ventral do pescoço, dispondo-se de forma linear e paralela, desde a décima primeira até a décima quinta vértebra cervical, relacionando-se com o nervo vago e veia jugular. $\mathrm{O}$ número de lobos variou de um a três e um ou dois, para os antímeros direito e esquerdo respectivamente. A média das dimensões dos lobos foram 2,09 $\times 0,71 \times 0,41 \mathrm{~cm}$ para o lobo cranial, $0,69 \times 0,38 \times 0,27 \mathrm{~cm}$ para o lobo médio e $0,55 \times 0,33 \times 0,29 \mathrm{~cm}$ para o lobo caudal do antímero direito. No antímero esquerdo, foram registrados $2,14 \times 0,71 \times 0,37 \mathrm{~cm}$ para o lobo cranial e $0,60 \times 0,31 \times 0,22 \mathrm{~cm}$ para o lobo caudal. A morfologia e biometria do timo apresentaram comportamentos bem definidos, possuindo características comuns quanto à holotopia, esqueletopia, sintopia e idiotopia, que deixaram caracterizada a espécie objeto de nosso estudo.

Palavras-chave: Anatomia; Aves; Região cervical; Sintopia

\section{Abstract}

Morphology and biometry of the thymus in ostrich (Sthruthio camelus). This work studied the anatomy of the thymus in 30 young ostriches (13 males and 17 females, 10 to 20 days old) that were fixed in an aqueous solution of $10 \%$ formaldehyde. Chains of thymic lobes, arranged linearly and parallel, were located in the third distal part of the ventral region of the neck from the eleventh to the fifteenth cervical vertebra, which relates to the vagus nerve and jugular vein. The number of lobes varied from 1 to 3 and 1 to 2 in the right and left 
antimeres, respectively. In the right antimere, the average dimensions of the lobes were $2.09 \times 0.71 \times 0.41 \mathrm{~cm}$ for the cranial lobe, $0.69 \times 0.38 \times 0.27 \mathrm{~cm}$ for the middle lobe and $55 \times 0.33 \times 0.29 \mathrm{~cm}$ for the caudal lobe. In the left antimere, the average dimensions were $2.14 \times 0.71 \times 0.37 \mathrm{~cm}$ for the cranial lobe and $0.60 \times 0.31 \times 0.22$ $\mathrm{cm}$ for the caudal lobe. The morphology and thymus biometrics showed well-defined traits, having common attributes, such as holotopy, skelotopy, syntopy and idiotopy, which characterized the species of this study.

Key words: Anatomy; Birds; Cervical region; Syntopy

\section{Introdução}

Os avestruzes pertencem ao grupo das ratitas, cuja etimologia remonta à palavra latina "ratis", que por sua vez significa um tipo de barco com o fundo chato, desprovido de quilha. Vivem cerca de 60 a 70 anos, podendo chegar a 80 anos na região da África do Sul (CARRER et al., 2004).

Dados morfológicos, como tamanho e topografia, podem ser utilizados como referências para a avaliação da imunocompetência individual, sendo que o tamanho do timo é um excelente indicador do estado de saúde das aves (MARÍN et al., 2004).

Hohn (1947) afirma que os timos de patos e gansos domésticos assemelham-se àqueles das espécies selvagens nas quais há tipicamente cinco lobos, sendo o mais cranial maior que os precedentes.

Hohn (1961) também afirma que a involução do timo das aves ocorre por ocasião da maturidade sexual, entretanto, uma nova hipertrofia e recuperação da arquitetura microscópica são verificadas durante algumas semanas após o primeiro e possivelmente último ciclo sexual. Acrescenta ainda que geralmente há alguma evidência de involução após algumas formas de estresse.

Tratadistas clássicos reportam-se ao timo das aves de maneira genérica, assim como Schwarze e Schröder (1972), Nickel et al. (1977), Kendall (1980), Getty (1986) e Baumel (1993), que descrevem o timo de galináceos como um órgão par, alargado e muito vascularizado, que se estende ao longo do pescoço juntamente com a veia jugular e nervo vago, até o tórax, sendo indiviso nas aves jovens e dividido em lobos em aves adultas. Getty (1986) ainda descreve que a glândula tireoide pode penetrar no tecido do timo.

Sem especificar linhagens de aves ou o antímero no qual os lobos se alojam, Schwarze e Schröder (1972) e
Getty (1986) descreveram de três a oito lobos. Nickel et al. (1977) descreveram de seis a oito lobos, acrescentando que estes apresentavam tamanhos variados. Kendall (1980) relata sete lobos para cada antímero, e que aqueles apresentavam tamanhos similares.

Scala et al. (1984), ao descreverem o timo de patos, admitiram que este é constituído de lobos que são, por sua morfologia, considerados entidades anatômicas isoladas, que ocupam grande extensão do pescoço.

Pesquisando os lobos tímicos em aves da linhagem Avian Farms, Santana et al. (2000) observaram uma variação de três a seis lobos para o antímero direito e de dois a nove lobos para o esquerdo.

Em aves da linhagem Label Rouge, Silva et al. (2001) encontraram de dois a seis lobos para o antímero direito, sendo que a frequência foi de forma crescente assim descrita: em 6,66\% ocorreram dois e seis lobos; em $23,33 \%$, quatro lobos; em $30 \%$, três lobos; e em 33,33\%, cinco lobos. Para o antímero esquerdo, relataram variação de um a cinco lobos descritos da mesma forma: em $10 \%$ das observações encontraram cinco lobos; em $16,66 \%$, quatro lobos; em $20 \%$, dois lobos; e, finalmente, em $26,66 \%$, um e três lobos. Destacaram também que em quatro das 30 aves analisadas (13,33\%), a glândula tireoide apresentava-se envolvida por um lobo tímico.

Em 30 galinhas da linhagem NPK, Santana et al. (2001) descreveram a presença de lobos tímicos cervicais, dispostos lateralmente ao pescoço em ambos os antímeros, e lobos tímicos toracoabdominais encontrados em apenas três aves, posicionados no antímero esquerdo. Os lobos tímicos variaram de um a sete e três a sete, nos antímeros direito e esquerdo, respectivamente.

Lima et al. (2004), analisando a morfologia do timo em patos (Anas platyrhynchos), descreveram-no formado por cadeias de lobos direita e esquerda localizadas ao 
longo do terço médio dos plexos vásculo-nervosos do pescoço, apresentando cada lobo uma forma irregular. A média do número de lobos foi de 11,5 por antímero. Com relação à morfometria, relatam que quanto maior o número de lobos, menor será o seu comprimento, espessura e largura.

Santana et al. (2004), analisando a espécie mutum (Crax fasciolata), descreveram que o timo ocupa posição dorsolateral aos plexos vásculo-nervosos do pescoço, apresentando lobos de formato irregular, com média de seis lobos por antímero.

Santana et al. (2007), em seus trabalhos com galinhas da Angola (Numidea meleagris galeata), afirmam que ficou caracterizada a presença de lobos tímicos cervicais, posicionados em ambos os antímeros do pescoço, em número variável de sete a 22 com maior predominância de sete lobos para o antímero direito e seis para o esquerdo, com comprimento e largura média de $1,12 \mathrm{~cm}$ e $0,30 \mathrm{~cm}$, respectivamente, para os lobos cervicais direitos e $1,27 \mathrm{~cm} \mathrm{e} 0,34 \mathrm{~cm}$ para os esquerdos, estando dispostos, preferencialmente, dorsolateral ao feixe vásculo-nervoso do pescoço.

Pereira et al. (2008) concluíram, em seus estudos com aves da linhagem Hubbard, que o número de lobos no antímero direito variava de quatro a 13, e para o esquerdo, de três a 11. Esses lobos localizaram-se desde a primeira vértebra cervical, até o limite com a cavidade toracoabdominal em íntima relação com a veia jugular e nervo vago.

Em galináceos da linhagem Cobb 500, as cadeias de lobos tímicos foram apreciadas por Birck et al. (2008). Estes constataram que se encontravam paralelos ao feixe vásculo-nervoso em número de três a cinco lobos, com predomínio do antímero esquerdo.

O timo em galináceos Bovans goldline foi descrito por Miranda et al. (2008), que encontraram o órgão disposto linear, paralelo e lateralmente na região cervical, relacionando-se com a artéria comum do nervo vago e com a veia jugular, apresentando um número de dois a seis lobos para cada antímero com formas e tamanhos variados.

Descrevendo o timo em galináceos da linhagem Paraíso Pedrês, Santana et al. (2008) relataram que as lobulações tímicas apresentaram uma variação de três a oito e de dois a 10 lobos respectivamente para os antímeros direito e esquerdo. Observaram também que o referido órgão localiza-se desde a fúrcula até próximo à mandíbula, na região cervical, dorsolateral ao plexo vásculo-nervoso do pescoço.

Em galináceos da linhagem Máster Gris Cou Plumé, Lima et al. (2009) descreveram topograficamente o timo e afirmaram que este se alojava nas regiões cervical e torácica e relataram de cinco a 11 e de quatro a nove lobos, respectivamente, nos antímeros esquerdo e direito, acrescentando que o referido órgão apresentava morfologias variadas.

Arantes et al. (2011) concluíram que, em galinhas caipiras (Gallus galllus), os lobos tímicos na região cervical, em ambos os antímeros, são dispostos dorsolateralmente ao feixe vásculo-nervoso, e foi registrada uma variação de nove a 15, assim distribuídos: o antímero direito mostrou-se com cinco a oito lobos, e o esquerdo com quatro a sete.

Em marrecos (Anas platyrhynchos platyrhynchos), Resende et al. (2012) constataram que os lobos tímicos estão presentes na região cervical, dispostos em cadeias laterais, em ambos os antímeros, demonstrando íntima relação com os nervos vagos, as veias jugulares e as artérias comuns dos nervos vagos, e apresentando formas e tamanhos variados. O número de lobos tímicos variou de três a cinco no antímero direito e de dois a cinco no esquerdo. Esses autores notaram que em algumas aves o timo apresentou um tamanho bastante reduzido.

Em estudo conduzido por Vianna et al. (2012), foram analisados 28 Gallus gallus domesticus da linhagem Red Bro Cou Nu. Os autores relataram que no antímero direito cada cadeia tímica apresentou de dois a 11 lobos tímicos, com maior frequência de oito lobos, e no antímero esquerdo, de um a oito lobos tímicos, sendo a maior frequência de cinco lobos.

Em 19 exemplares de araras de três gêneros diferentes, sendo 12 exemplares de Ara arauna, cinco de Ara chloropterus e dois de Anodorhynchus hyacinthinus, não foram encontrados lobos cervicais em dois casos (10,5\%) e lobos celomáticos em 11 (57,9\%). Os lobos apresentaram formatos alongados em 52,6\% dos casos, 
arredondados em $21,1 \%$ e ovalados em $15,8 \%$. O número médio de lobos por antímero foi de 5 , o tamanho médio foi de $0,49 \mathrm{~cm}$ de comprimento, $0,12 \mathrm{~cm}$ de largura, $0,05 \mathrm{~cm}$ de espessura e peso médio de $0,076 \mathrm{~g}$ (LIMA et al., 2013).

O presente trabalho espera contribuir para um melhor conhecimento da anatomia dessas glândulas na espécie Struthio camelus.

\section{Material e Métodos}

Foram utilizados neste trabalho 30 filhotes de avestruzes, 13 machos e 17 fêmeas com idade variando entre 10 a 20 dias, doadas, após morte natural, por criatórios credenciados pelo IBAMA do estado de Minas Gerais.

O trabalho foi submetido à análise do Comitê de Ética na Utilização de Animais da Universidade Federal de Uberlândia e obteve o parecer favorável (protocolo CEUA/UFU 046/15).

Logo após o óbito, o peso de cada animal foi determinado, com o uso de uma balança eletrônica pesadora e contadora da marca Digitron, e, em seguida, foram congelados e transportados para o Laboratório de Anatomia dos Animais Domésticos da Universidade Federal de Uberlândia, Uberlândia/ Minas Gerais.

Após descongelamento em água corrente, os avestruzes tiveram o sistema arterial preparado mediante isolamento e canulação da artéria isquiática direita, em sentido cranial no corpo e distal no membro, com cânula de polietileno, de calibre compatível ao diâmetro do vaso, e posterior injeção de solução aquosa, de Neoprene Látex “450” a 50\% (Du Pont do Brasil S/A - Indústria Química), corada com pigmento específico (Globo S/A Tintas e Pigmentos), visando à exploração dos vasos arteriais em futuras pesquisas. Posteriormente ao preenchimento dos vasos arteriais, as peças permaneceram em descanso por um período mínimo de $4 \mathrm{~h}$, mergulhadas em água gelada de 5 a $10^{\circ} \mathrm{C}$, para coagulação do látex.

Após o período de descanso, cada ave foi fixada em solução aquosa de formol a 10\% (LABSYNTH Produtos para Laboratório Ltda), por meio de injeção subcutânea, intramuscular profunda e intracavitária. Em seguida, as peças foram imersas e mantidas na mesma solução.

Em uma etapa seguinte, uma semana após a fixação, com o auxílio de bisturi, lâminas, tesouras e pinças anatômicas adequadas, se procedeu à dissecação. Para isso foi feita a exposição da região ocupada pelo timo. Mediante incisão mediana ventral da pele da região cervical, seguida da divulsão do tecido conjuntivo subcutâneo e do afastamento dos músculos, os lobos cervicais direito e esquerdo foram identificados e, através de secção dos músculos peitorais e dos ossos clavícula, coracoide e esterno, todo o conjunto de estruturas anatômicas da parede torácica foi rebatido caudalmente para se verificar a presença de timo torácico.

Foram realizadas mensurações de cada lobo tímico com o auxílio de paquímetro digital, sendo determinadas as dimensões: de comprimento, largura e espessura.

Todos os dados arregimentados foram registrados em esquemas confeccionados de todas as preparações e obtidas fotografias. Os termos anatômicos foram baseados na Nomina Anatomica Avium, proposta por Baumel (1993).

\section{Resultados}

O peso de cada avestruz foi aferido, e o peso médio variou de 1008 a $2280 \mathrm{~g}$ com mediana de $1838,53 \mathrm{~g}$.

Quanto à holotopia, essas cadeias localizaram-se no terço distal da região cervical ventral do pescoço, dispondo-se de forma linear e paralela, sendo que sua porção cranial tende a afastar-se do plano sagital mediano, assumindo posição lateral, enquanto sua porção caudal aproxima-se do plano mencionado, assumindo assim posição medial.

Sua esqueletopia pode ser descrita desde a décima primeira vértebra cervical, até o limite com a cavidade celomática, próximo à junção entre a décima quinta vértebra cervical e a primeira torácica, nunca ultrapassando caudalmente esse limite.

A sintopia mostra íntima relação com o nervo vago e com a veia jugular externa em sua disposição linear 
ao longo do pescoço e com a glândula tireoide no seu limite caudal. Abundante tecido conjuntivo frouxo, tecido adiposo, músculos adjacentes e as estruturas de sua relação de sintopia garantem a eficácia de sua estática visceral (Figuras 1 e 2).

Quanto à idiotopia, seus lobos apresentaram-se individualizados, podendo apresentar pequenas fissuras em sua superfície. O número de lobos tímicos no antímero direito variava de um a três, mais particularmente: um lobo 10 vezes $(33,33 \%)$; dois lobos 18 vezes $(60 \%)$; e três lobos duas vezes $(6,66 \%)$, com média de 1,73 lobos. No antímero esquerdo foram encontrados de um a dois lobos, mais especificamente: um lobo 14 vezes $(46,66 \%)$ e dois lobos 16 vezes $(53,33 \%)$, com média de 1,53 lobos (Tabelas 1 e 2).

FIGURA 1: Região lateral direita do pescoço de avestruz (Struttio camellus), evidenciando a relação de topografia dos lobos tímicos (D) com a artéria comum do nervo vago direita (C), veia jugular direita (B) e nervo vago direito (A). O tecido adiposo (E) reduzido do campo visual. Fonte: Arquivo pessoal do autor Cheston Cesar Honorato Pereira.

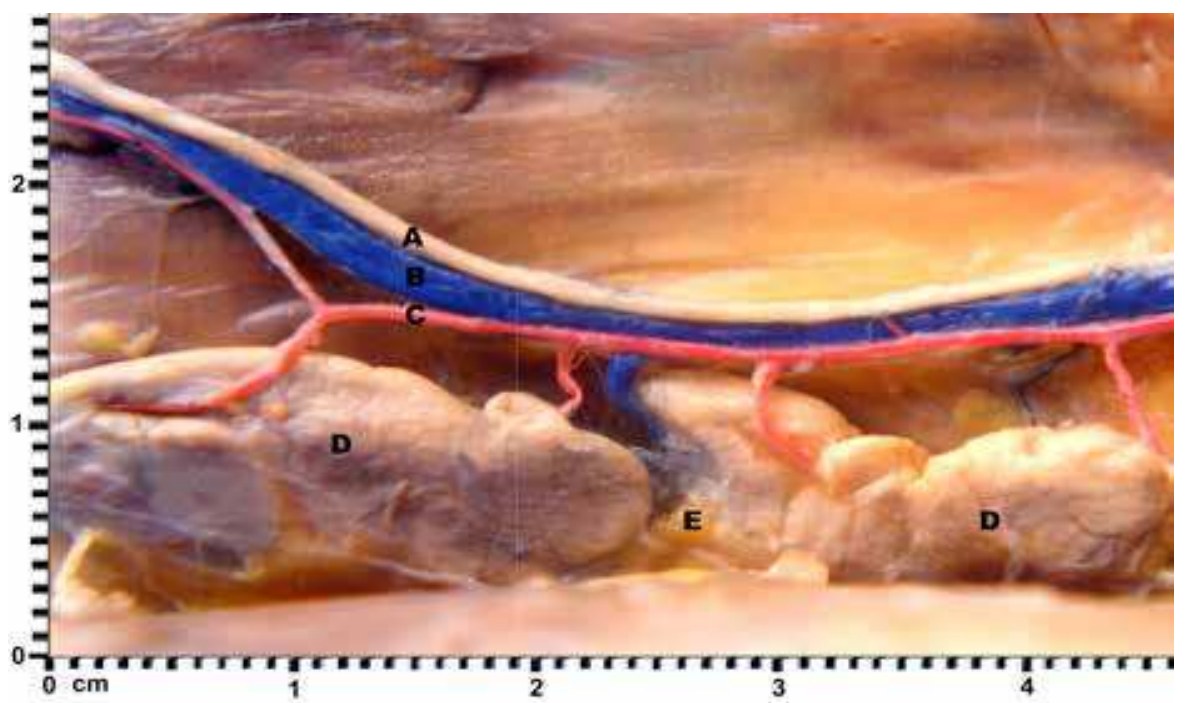

FIGURA 2: Região lateral direita do pescoço de avestruz (Struttio camellus), evidenciando a relação de topografia dos lobos tímicos (A) com a artéria comum do nervo vago direita (B), nervo vago direito (C), tireoide direita (D) e esôfago (E). Fonte: Arquivo pessoal do autor Cheston Cesar Honorato Pereira.

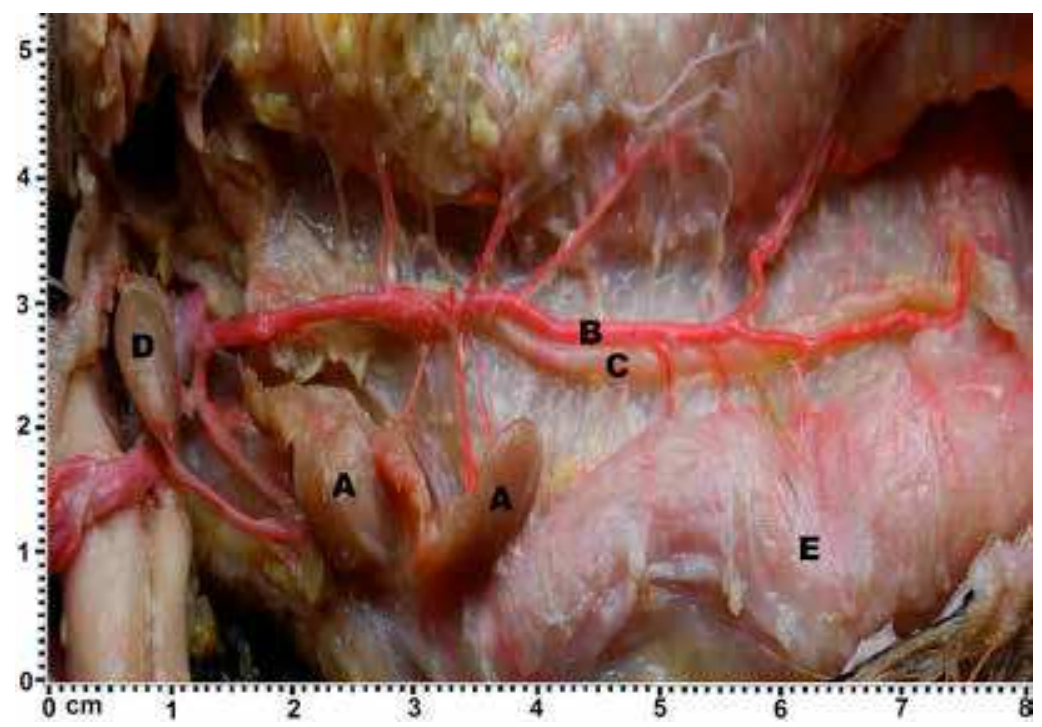


TABELA 1: Representação do número de lobos tímicos, número de ocorrências e porcentagem independentes do antímero, Uberlândia, MG -2015 .

\begin{tabular}{ccc}
\hline Número de lobos & Número de aves & $\mathbf{\%}$ \\
\hline 2 & 6 & 20 \\
3 & 11 & 36,66 \\
4 & 12 & 40 \\
5 & 1 & 3,33 \\
\hline & 30 & 99,99 \\
\hline
\end{tabular}

TABELA 2: Representação do número de lobos tímicos, número de ocorrências e porcentagens de acordo com o antímero, Uberlândia, MG 2015.

\begin{tabular}{|c|c|c|c|c|c|}
\hline \multirow{2}{*}{$\begin{array}{l}\text { Número } \\
\text { de lobos }\end{array}$} & \multicolumn{2}{|c|}{$\begin{array}{c}\text { Antímero } \\
\text { direito }\end{array}$} & \multirow{2}{*}{$\begin{array}{l}\text { Número } \\
\text { de lobos }\end{array}$} & \multicolumn{2}{|c|}{$\begin{array}{l}\text { Antímero } \\
\text { esquerdo }\end{array}$} \\
\hline & $\begin{array}{l}\text { Número } \\
\text { de aves }\end{array}$ & $\%$ & & $\begin{array}{l}\text { Número } \\
\text { de aves }\end{array}$ & $\%$ \\
\hline 1 & 10 & 33,33 & 1 & 14 & 46,66 \\
\hline 2 & 18 & 60 & 2 & 16 & 53,33 \\
\hline 3 & 2 & 6,66 & & & \\
\hline & 30 & 99,99 & & 30 & 99,99 \\
\hline
\end{tabular}

Nas 30 aves estudadas, não se identificou a presença do referido órgão na cavidade toracoabdominal ou qualquer aderência de seu parênquima às glândulas tireoides.

Em termos biométricos para os lobos tímicos do antímero direito, a medida média de comprimento, largura e espessura dos lobos dos animais que possuem apenas um lobo foi de 2,46 x 0,98 x 0,54 cm. Para os animais que possuem dois lobos as medidas médias para os lobos craniais foram de $1,94 \times 0,57 \times 0,33 \mathrm{~cm}$ e para os lobos caudais de $0,67 \times 0,36 \times 0,26 \mathrm{~cm}$. Finalmente, para aqueles que possuem três lobos, as médias foram de $1,64 \times 0,59 \times 0,39 \mathrm{~cm}$ para os lobos craniais, $0,89 \times 0,56 \times 0,33 \mathrm{~cm}$ para os lobos médios e $0,55 \times 0,33 \times 0,29 \mathrm{~cm}$ para os lobos caudais (Tabela 3 ).

As dimensões dos lobos do antímero esquerdo foram da mesma forma determinadas, sendo $2,42 \times 0,93 \times 0,46 \mathrm{~cm}$ para aqueles animais que apresentaram somente um lobo. Para aqueles que possuem dois lobos as dimensões foram de $1,89 \times 0,51 \times 0,29 \mathrm{~cm}$ para os lobos craniais e de $0,60 \times 0,31 \times 0,22 \mathrm{~cm}$ para os lobos caudais (Tabela 4 ).

TABELA 3: Dimensões dos lobos tímicos do antímero direito de avestruz (Struthio camelus), representando as medidas de comprimento (C), largura (L) e espessura (E) de cada lobo. Uberlândia, MG - 2015.

\begin{tabular}{|c|c|c|c|c|c|c|c|c|c|}
\hline \multirow{2}{*}{ Identificação } & \multicolumn{3}{|c|}{ Lobo cranial } & \multicolumn{3}{|c|}{ Lobo médio } & \multicolumn{3}{|c|}{ Lobo caudal } \\
\hline & C & $\mathbf{L}$ & $\mathbf{E}$ & $\mathrm{C}$ & $\mathbf{L}$ & $\mathbf{E}$ & $\mathrm{C}$ & $\mathbf{L}$ & $\mathbf{E}$ \\
\hline & 2,43 & 0,98 & 0,53 & & & & & & \\
\hline & 2,52 & 1,00 & 0,63 & & & & & & \\
\hline & 2,00 & 0,65 & 0,40 & 0,81 & 0,48 & 0,31 & & & \\
\hline & 2,11 & 0,68 & 0,42 & 0,88 & 0,49 & 0,30 & & & \\
\hline & 2,48 & 0,99 & 0,55 & & & & & & \\
\hline & 2,39 & 0,96 & 0,48 & & & & & & \\
\hline & 1,98 & 0,63 & 0,38 & 0,78 & 0,45 & 0,29 & & & \\
\hline & 1,95 & 0,59 & 0,33 & 0,70 & 0,39 & 0,28 & & & \\
\hline & 2,01 & 0,66 & 0,38 & 0,79 & 0,45 & 0,28 & & & \\
\hline & 1,90 & 0,56 & 0,30 & 0,65 & 0,30 & 0,25 & & & \\
\hline & 1,60 & 0,58 & 0,37 & 0,86 & 0,51 & 0,30 & 0,50 & 0,32 & 0,28 \\
\hline & 1,96 & 0,61 & 0,31 & 0,66 & 0,32 & 0,22 & & & \\
\hline & 1,68 & 0,60 & 0,40 & 0,92 & 0,61 & 0,36 & 0,60 & 0,34 & 0,30 \\
\hline & 1,91 & 0,58 & 0,33 & 0,62 & 0,29 & 0,24 & & & \\
\hline & 2,55 & 1,01 & 0,60 & & & & & & \\
\hline & 1,85 & 0,48 & 0,26 & 0,52 & 0,25 & 0,23 & & & \\
\hline & 2,44 & 0,96 & 0,51 & & & & & & \\
\hline & 2,02 & 0,67 & 0,40 & 0,80 & 0,46 & 0,30 & & & \\
\hline & 1,84 & 0,46 & 0,26 & 0,50 & 0,24 & 0,23 & & & \\
\hline & 1,83 & 0,44 & 0,25 & 0,51 & 0,23 & 0,23 & & & \\
\hline
\end{tabular}




\begin{tabular}{|c|c|c|c|c|c|c|c|c|c|}
\hline & 1,97 & 0,60 & 0,32 & 0,69 & 0,40 & 0,26 & & & \\
\hline & 2,02 & 0,68 & 0,38 & 0,80 & 0,47 & 0,31 & & & \\
\hline & 1,78 & 0,39 & 0,25 & 0,48 & 0,22 & 0,20 & & & \\
\hline & 2,50 & 1,01 & 0,60 & & & & & & \\
\hline & 2,48 & 1,02 & 0,62 & & & & & & \\
\hline & 1,79 & 0,36 & 0,26 & 0,45 & 0,21 & 0,20 & & & \\
\hline & 2,01 & 0,65 & 0,38 & 0,78 & 0,44 & 0,29 & & & \\
\hline & 1,98 & 0,62 & 0,34 & 0,68 & 0.38 & 0,25 & & & \\
\hline & 2,35 & 0,90 & 0,43 & & & & & & \\
\hline & 2,43 & 0,97 & 0,48 & & & & & & \\
\hline Média & 2,09 & 0,71 & 0,41 & 0,69 & 0,38 & 0,27 & 0,55 & 0,33 & 0,29 \\
\hline Máximo & 2,55 & 1,02 & 0,63 & 0,92 & 0,61 & 0,36 & 0,6 & 0,34 & 0,30 \\
\hline Mínimo & 1,60 & 0,36 & 0,25 & 0,45 & 0,21 & 0,20 & 0,5 & 0,32 & 0,28 \\
\hline
\end{tabular}

TABELA 4: Dimensões dos lobos tímicos do antímero esquerdo de avestruz (Struthio camelus), representando as medidas de comprimento (C), largura (L) e espessura (E) de cada lobo. Uberlândia, MG - 2015.

\begin{tabular}{|c|c|c|c|c|c|c|}
\hline \multirow{2}{*}{ Identificação } & \multicolumn{3}{|c|}{ Lobo cranial } & \multicolumn{3}{|c|}{ Lobo médio } \\
\hline & $\mathbf{C}$ & $\mathbf{L}$ & $\mathbf{E}$ & $\mathrm{C}$ & $\mathbf{L}$ & $\mathbf{E}$ \\
\hline & 2,38 & 0,87 & 0,48 & 0,00 & 0,00 & 0,00 \\
\hline & 2,01 & 0,66 & 0,41 & 0,83 & 0,46 & 0,32 \\
\hline & 1,82 & 0,45 & 0,23 & 0,51 & 0,22 & 0,23 \\
\hline & 1,86 & 0,44 & 0,23 & 0,52 & 0,21 & 0,21 \\
\hline & 2,39 & 0,91 & 0,44 & & & \\
\hline & 2,22 & 0,76 & 0,28 & & & \\
\hline & 1,88 & 0,53 & 0,32 & 0,68 & 0,35 & 0,22 \\
\hline & 1,95 & 0,59 & 0,33 & 0,70 & 0,39 & 0,28 \\
\hline & 2,37 & 0,88 & 0,39 & & & \\
\hline & 1,88 & 0,51 & 0,26 & 0,55 & 0,27 & 0,20 \\
\hline & 2,45 & 0,98 & 0,38 & & & \\
\hline & 1,90 & 0,58 & 0,29 & 0,55 & 0,27 & 0,19 \\
\hline & 2,00 & 0,65 & 0,32 & 0,77 & 0,44 & 0,22 \\
\hline & 2,48 & 0,95 & 0,49 & & & \\
\hline & 2,56 & 1,01 & 0,58 & & & \\
\hline & 1,81 & 0,42 & 0,24 & 0,50 & 0,31 & 0,20 \\
\hline & 2,01 & 0,66 & 0,32 & 0,75 & 0,38 & 0,22 \\
\hline & 2,00 & 0,64 & 0,36 & 0,76 & 0,38 & 0,28 \\
\hline & 1,82 & 0,40 & 0,25 & 0,48 & 0,22 & 0,23 \\
\hline & 2,45 & 0,91 & 0,52 & & & \\
\hline & 1,78 & 0,34 & 0,22 & 0,41 & 0,23 & 0,21 \\
\hline & 2,52 & 1,02 & 0,61 & & & \\
\hline & 1,76 & 0,34 & 0,23 & 0,40 & 0,20 & 0,16 \\
\hline & 1,80 & 0,37 & 0,28 & 0,47 & 0,23 & 0,19 \\
\hline & 2,00 & 0,62 & 0,38 & 0,77 & 0,42 & 0,22 \\
\hline & 2,37 & 0,92 & 0,41 & & & \\
\hline & 2,48 & 0,99 & 0,49 & & & \\
\hline & 2,52 & 0,99 & 0,53 & & & \\
\hline & 2,28 & 0,87 & 0,37 & & & \\
\hline & 2,38 & 0,90 & 0,41 & & & \\
\hline Média & 2,14 & 0,71 & 0,37 & 0,60 & 0,31 & 0,22 \\
\hline Maior & 2,56 & 1,02 & 0,61 & 0,83 & 0,46 & 0,32 \\
\hline Menor & 1,76 & 0,34 & 0,22 & 0,40 & 0,20 & 0,16 \\
\hline
\end{tabular}


Em termos genéricos, as médias das dimensões foram de $2,09 \times 0,71 \times 0,41 \mathrm{~cm}$ para o lobo cranial, de $0,69 \times 0,38 \times 0,27 \mathrm{~cm}$ para o lobo médio e de $0,55 \times 0,33 \times 0,29 \mathrm{~cm}$ para o lobo caudal do antímero direito. Para o antímero esquerdo, encontramos $2,14 \times 0,71 \times 0,37 \mathrm{~cm}$ para os lobos craniais e $0,60 \times 0,31 \times 0,22 \mathrm{~cm}$ para os lobos caudais.

\section{Discussão}

Com relação à holotopia, que descreve as cadeias de lobos tímicos localizadas no terço distal da região cervical ventral do pescoço, dispondo-se de forma linear e paralela, concordamos com os achados de Schwarze e Schröder (1972), Nickel et al. (1977), Kendall (1980), Getty (1986), Baumel (1993), Scala et al. (1984), Santana et al. (2000; 2001; 2004; 2007; 2008), Silva et al. (2001), Lima et al. (2004; 2009; 2013), Pereira et al. (2008), Birck et al. (2008), Miranda et al. (2008), Arantes et al. (2011), Resende et al. (2012) e Vianna et al. (2012). De outra forma, Santana et al. (2001) e Lima et al. $(2009 ; 2013)$ encontraram lobos tímicos dentro da cavidade toracoabdominal, fato este não ocorrido nos avestruzes deste estudo.

A esqueletopia aqui é detalhada, em seus limites cranial e caudal. Somente Lima et al. (2004) restringemse à sua relação caudal com os ossos claviculares. Pereira et al. (2008) citaram sua relação cranial com a primeira vértebra cervical e Arantes et al. (2011), com a terceira vértebra cervical. Os autores estabeleceram como limite cranial os ossos mandibulares, e como limite caudal, os claviculares.

Quanto à sua sintopia, assim como foi descrito, sua relação com o feixe vásculo-nervoso é citada por Schwarze e Schröder (1972), Nickel et al. (1977), Kendall (1980), Getty (1986), Santana et al. (2004; 2007) e Birck et al. (2008).

Schwarze e Schröder (1972), Nickel et al. (1977), Getty (1986) e Kendall (1980) relataram ainda que em aves jovens o timo apresenta-se indiviso, fato este não registrado para os avestruzes que, apesar de serem jovens, apresentaram os lobos tímicos em pouco número, mas perfeitamente individualizados.
Quanto à relação entre os vasos arteriais que nutrem o parênquima tímico de cada lobo, não houve a formação de um ponto comum de penetração, como citado por Scala et al. (1984), Pereira et al. (1998), Santana et al. (2000; 2001; 2008) e Vianna et al. (2012), que também não relataram a existência de um hilo tímico, coadunando com nossa pesquisa.

Quanto à idiotopia, os resultados desta pesquisa estão de acordo com as pesquisas de Nickel et al. (1977), Getty (1986), Miranda et al. (2008), Pereira et al. (2008), Arantes et al. (2011) e Lima et al. (2013), sendo que o lobo cranial é sempre maior, discordando de Hohn (1947) e Resende et al. (2012). A superfície dos lobos tímicos dos avestruzes apresentou pequenas fissuras, mas que não definem uma separação total do tecido tímico a ponto de formarem novos lobos.

O número de lobos tímicos no antímero direito variou de um a três. Apresentaram, portanto, um número reduzido de lobos quando comparados com os achados dos autores que pesquisaram galináceos. Esses apontamentos, conforme elucidado por Hohn (1947) e confirmados por Resende et al. (2012), nos levam a conjecturar que o timo de avestruzes provavelmente tenha correspondências morfológicas com aqueles das espécies selvagens, nas quais verifica-se de dois a cinco lobos, conforme relatado por Resende et al. (2012) em marrecos ou uma mediana de seis lobos, como exposto por Santana et al. (2004) em mutum de penacho.

A glândula tireoide envolta por tecido tímico foi descrita por Getty (1986), Silva et al. (2001), Lima et al. (2009) e Arantes et al. (2011). Em nossa pesquisa, nas 30 aves estudadas, não se identificou aderência do timo às glândulas tireoides. Essas ocorrências podem ser características próprias da espécie de ave analisada.

Somente Santana et al. (2007) e Lima et al. (2013) pesquisaram a morfometria do timo, sendo que os primeiros trataram apenas do comprimento e largura, e os demais autores, assim como em nossa pesquisa, relacionaram comprimento, largura e espessura. De acordo com as medidas encontradas por esses autores, podemos dizer que as dimensões dos lobos tímicos de avestruzes são superiores àquelas encontradas por eles. Isso se torna claro devido à 
grande massa corporal exibida pelos animais objetos de estudo neste trabalho.

Com relação à posição de cada lobo e a variação de tamanho, Baumel (1993), Lima et al. (2004), Santana et al. (2007; 2008) e Arantes (2011) relataram que a posição ocupada por eles e o seu tamanho são variáveis, como demonstrado nos avestruzes, os lobos menores tendem a ocupar posições mais ventrolaterais em relação ao plexo vásculo-nervoso, enquanto lobos maiores tendem a ocupar uma posição mais dorsolateral (SANTANA et al., 2008). Além dessas observações, verificamos que, em sua porção caudal, os lobos do antímero direito tendem a se aproximar do plano sagital mediano, enquanto em sua porção cranial estes tendem a se afastar desse plano, tomando uma posição mais lateral.

Podemos concluir que em avestruzes foram encontradas características comuns que dizem respeito aos achados de holotopia, esqueletopia, sintopia e idiotopia, ou seja, que a posição no corpo, as suas relações com o esqueleto, a relação com órgãos vizinhos e dos lobos tímicos entre si são comuns a todos os animais dissecados, fato este que deixou caracterizado o timo da espécie objeto de nosso estudo.

\section{Referências}

ARANTES, R. C.; HONORATO, A. G. de O.; SEVERINO, R. S.; FERREIRA, D.; PEREIRA, C. C. H. Vascularização arterial do timo em galinha caipira (Gallus gallus). PUBVET, Londrina, v. 5, n. 14, p. 1-15, 2011.

BAUMEL, J. J. Handbook of avian anatomy: Nomina Anatomica Avium. 2. ed. Cambridge: Nuttall Ornithological Club, 1993. 779 p.

BIRCK, A. J.; RESENDE, H. R. A.; FILADELPHO, A. L.; BIRCK, N. M. M. Suprimento arterial do timo de aves (Gallus gallus) da linhagem cobb 500. Revista Científica Eletrônica de Medicina Veterinária, Garça, v. 6, n. 11, p. 30-37, 2008.

CARRER, C. C.; ELMÔR, R. A.; KONFELD, M. E.; CARVALHO, M. C. A criação de avestruz: guia completo de A a Z. Pirassununga: C.C. Carrer, 2004. 255 p.

GETTY, R. Sisson/Grossman anatomia dos animais domésticos. 5. ed. Rio de Janeiro: Guanabara Koogan, 1986. 2048 p.

HOHN, E. O. Seasonal cyclical changes in the thymus of the Mallard. Journal Experimental Biology, Cambridge, v. 24, p. 184-191, 1947.

HOHN, E. O. Endocrine glands, thymus and pineal body. In: MARSHALL, A. S. (Ed.). Biology and comparative physiology of birds. Vol. II. New York: Academic Press. 1961. p. 87-114.
KENDALL, M. D. Avian thymus gland. Developmental and Comparative Immunology, London, v. 4, n. 1, p. 191-209, 1980.

LIMA, E. M. M. de; BRAVO, M. de O.; VIANNA, A. R. da C. B.; CARNEIRO E SILVA, F. O.; SEVERINO, S. R.; DRUMMOND, S. S.; SANTANA, M. I. S. Suprimento vascular arterial do timo em aves (Gallus gallus) da linhagem Máster Gris Cou Plumé. Biotemas, Florianópolis, v. 22, n. 3, p. 171-176, 2009.

LIMA, R. Z.; GONÇALVES, E. S.; SANTANA, M. I.; LIMA, E. M. M.; SILVA, F. O. C.; SEVERINO, R. S.; DRUMMOND, S. S.; ARMANDO, A. P. R. N. Morfologia e biometria do timo em araras dos gêneros Ara e Anodorhyncus. Arquivos Brasileiros de Medicina Veterinária e Zootecnia, Belo Horizonte, v. 26, n. 1, p. 103-111, 2013.

LIMA, R. Z.; SANTANA, M. I.; DOS REIS, I. A. Morfologia e vascularização do timo em patos (Anas platyrhynchus). In: REUNIÃO DE INTEGRAÇÃO DA MORFOLOGIA PANAMERICANA/CONGRESSO BRASILEIRO DE ANATOMIA, 8/ XXI, 2004, Foz do Iguaçu. Anais... Foz do Iguaçu: UFPR, 2004. p. 12.

MARÍN, F. P.; NAVA, J.; MAVÁREZ, Y. Caracterización morfométrica de los organos linfoides en pollos de engorde de la línea Ross criados bajo condiciones de campo en el estado Zulia, Venezuela. Revista Científica, Maracaibo, v. 14, p. 217-225, 2004. MIRANDA, R. L.; SILVA, F. O. C.; SEVERINO, R. S.; DRUMMOND, S. S.; MENDONÇA, E. P.; SOLA, M. C.; JÚNIOR, W. S. Artéria do timo em aves (Gallus gallus) poedeiras da linhagem Bovans Goldline. Bioscience Journal, Uberlândia, v. 24, n. 4, p. 138-142, 2008.

NICKEL, R.; SCHUMMER, A.; SEIFERLE, E. Anatomy of the domestic birds. Berlin: Verlag Paul Parey, 1977. 202 p.

PEREIRA, C. C. H.; BOMBONATO, P. P.; CARNEIRO E SILVA, F. O.; HONORATO, A. das G. de O. Irrigação arterial do timo em aves (Gallus gallus domesticus) da linhagem Hubbard. Veterinária Notícias, Uberlândia, v. 14, n. 1, p. 41-47, 2008.

RESENDE, G. G. N.; FERREIRA, F. A.; SILVA, F. O. C.; VASCONCELOS, B. G.; IGLESIAS, L. P.; SANTOS, A. C.; MIGLINO, M. A. Suprimento vascular arterial do timo de marrecos (Anas platyrhynchos platyrhynchos). Revista Científica Eletrônica de Medicina Veterinária, Garça, v. 9, n. 18, p. 1-10, 2012.

SANTANA, M. I. S.; BOMBONATO, P. P.; ARAÚJO, L. V.; CASTELlO, A. P. F.; ESCANHOELA, T. B. Irrigação do timo em aves da linhagem Paraíso Pedrês (Gallus gallus). Arquivos Brasileiros de Medicina Veterinária e Zootecnia, São Paulo, v. 60, n. 2, p. 307-314, 2008.

SANTANA, M. I. S.; BOMBONATO, P. P.; CARNEIRO E SILVA, F. O.; BENEDICTO, H. G. Morfologia e morfometria do timo em galinhas de Angola (Numidea meleagris galeata). Revista Portuguesa de Ciências Veterinárias, Lisboa, v. 102, n. 561-562, p. 43-48, 2007.

SANTANA, M. I. S.; CARNEIRO E SILVA, F. O.; DRUMMOND, S. S.; SEVERINO, R. S.; BOMBONATO, P. P. Vascularização arterial do timo em aves (Gallus gallus domesticus) da linhagem NPK. Veterinária Notícias, Uberlândia, v. 7, n. 1, p. 13-19, 2001.

SANTANA, M. I. S.; CARNEIRO E SILVA, F. O.; DRUMMOND, S. S.; SEVERINO, R. S.; BOMBONATO, P. P.; SANTOS, A. L. Q. Vascularização arterial do timo em aves (Gallus gallus domesticus) 
da linhagem Avian Farms. Bioscience Journal, Uberlândia, v. 16, n. 2, p. 3-15, 2000.

SANTANA, M. I. S.; NETTO, A. P.; SILVA, F. O. C. Morfologia e irrigação do timo em mutuns (Crax fasciolata). In: REUNIÃO DE INTEGRAÇÃO DA MORFOLOGIA PAN-AMERICANA/ CONGRESSO BRASILEIRO DE ANATOMIA, 8/XXI, 2004, Foz do Iguaçu. Anais... Foz do Iguaçu: UFPR, 2004. p. 14.

SCALA, G.; LANGELlA, M.; BUDETTA, G. Sulla vascularizzazione del timo nell anatra. Bollettino della Società Italiana di Biología Sperimentale, Napoli, v. 60, n. 4, p. 701-706, 1984.
SCHWARZE, E.; SCHRÖDER, L. Compêndio de anatomia veterinária. Vol. 5. Zaragoza: Acribia, 1972. 212 p.

SILVA, F. O. C.; SEVERINO, R. S.; DRUMMOND, S. S.; PERISSOTO, D. O.; CAMPOS, D. B.; CAMPOS, A. B. Artérias do timo de aves (Gallus gallus) da linhagem Label Rouge. Bioscience Journal, Uberlândia, v. 17 n. 2, p. 115-123, 2001.

VIANNA, A. R. da C. B.; MELO, F. A. C. de; SANTANA, M. I. S.; BENEDICTO, H. G.; CARNEIRO E SILVA, F. O.; SEVERINO, R. S.; LIMA, E. M. M.; Macroscopic morphology and arterialvascular supply of thymus in fowls (Gallus gallus domesticus) of Red Bro Cou $\mathrm{Nu}$ lineage. Acta Scientiarum, Maringá, v. 34, n. 4, p. 457$462,2012$. 